\title{
LA AUTOEFICACIA Y SU RELACIÓN CON LA SALUD PSICOSOCIAL OCUPACIONAL EN MÉDICOS DE URGENCIAS HOSPITALARIAS
}

\section{SELF-EFFICACY AND ITS RELATIONSHIP WITH THE OCCUPATIONAL PSYCHOSOCIAL HEALTH IN DOCTORS OF HOSPITAL EMERGENCIES}

\section{MIGUEL BERNABÉ CASTAÑO}

Centro Universitario de la Defensa

Zaragoza, España.

mbernabe@unizar.es

\section{MAITE MARTÍN-ARAGÓN GELABERT}

martin-aragon@umh.es

\section{CARMEN TEROL CANTERO}

macarmen@umh.es

Departamento de Psicología de la Salud Universidad Miguel Hernández de Elche Alicante, España.

DOI: http://doi.org/10.29035/ucmaule.53.75

\section{RESUMEN}

En los últimos años, se ha prestado especial atención al papel de la autoeficacia sobre la salud ocupacional en diferentes profesiones, siendo escasos los trabajos en médicos. El objetivo del estudio es analizar la relación entre la autoeficacia y variables del bienestar y el malestar laboral, a partir de un modelo teórico previo. Para ello, se solicitó la participación voluntaria de los 117 médicos de los Servicios de Urgencias Hospitalarias (SUH) de Alicante, de los cuales el $54.8 \%$ eran hombres. La tasa de respuesta fue del $71.8 \%(n=84)$. Se administraron las escalas de Agota- miento Emocional y Cinismo del MBIGS y Vigor y Dedicación del UWES como medida de malestar y bienestar. La Escala de Autoeficacia General se administró para medir autoeficacia. Se realizan análisis descriptivos y análisis factorial confirmatorio. Los resultados confirman el modelo teórico propuesto, así como las relaciones entre variables. Las creencias de autoeficacia predicen el bienestar y malestar experimentado por los médicos de los SUH.

Palabras clave: médicos, Burnout, Engagement, autoeficacia. 


\section{ABSTRACT}

In recent years, special attention is paid to the role of self-efficacy on occupational health in different professions, with few studies on physicians. The aim of the study is to analyze the relationship between self-efficacy and well-being variables and labor unrest starting from a previous theoretical model. The sample was composed by 117 physicians of Hospital Emergency Services (HES) in Alicante, $54.8 \%$ male, composed the sample. The response rate was $71.8 \%$ ( $n=84)$. We administered Emotional Exhaustion and Cynicism Scales of
MBI-GS and Vigor and Dedication Scales of UWES as a measure of unrest and welfare. The General Self-Efficacy Scale was administered to measure self-efficacy. Descriptive analyzes are conducted and confirmatory factor analysis. The results confirm the theoretical model proposed and the relationships between variables. Self-efficacy beliefs predict wellbeing and discomfort experienced by HES physicians.

Key words: physicians, Burnout, Engagement, self-efficacy.

\section{INTRODUCCIÓN}

En las últimas décadas, ha surgido un interés renovado por el estudio de los procesos de Salutogénesis, que posibilitan alcanzar niveles máximos de ajuste biopsicosocial y que hacen referencia a cómo las personas manejan el estrés y continúan sintiéndose bien a pesar de las dificultades que afrontan (Antonovsky, 1987). Tal interés se inicia con Seligman y Csikszentmihalyi (2000) cuando acuñan el término de Psicología Positiva, la cual agrupa los estudios centrados en el análisis de las fortalezas humanas y el efecto de las emociones positivas en el comportamiento de la persona. Todo esto supone un giro en los enfoques tradicionales de la psicología, centrados principalmente en el estudio de variables como la ansiedad, la depresión o el sufrimiento (Seligman, 2008).

Esa tradición de enfoque también se observa en el ámbito de la salud ocupacional, pues la mayoría de las investigaciones generadas hasta el momento tienen su atención en aspectos como el estrés laboral y el Burnout.

Sin embargo, se ha propuesto una novedosa perspectiva que, bajo el término de Psicología Ocupacional Positiva, aborda el estudio de las variables que potencian la calidad de vida laboral y organizacional, así como las fortalezas de los 
trabajadores, la iniciativa personal y el funcionamiento organizacional saludable (Salanova, Martínez y Llorens, 2005; Lisbona y Palací, 2010). En este contexto surge también el constructo Engagement, referido a un estado motivacionalafectivo positivo con pleno sentido en el trabajo, caracterizado por aspectos como el vigor, dedicación y absorción (Schaufeli, Salanova, González-Romá y Bakker, 2002).

Sin querer cambiar la orientación de los estudios y para evitar caer en una visión incompleta de la salud, centrada exclusivamente en los aspectos novedosos y positivos en detrimento de los tradicionales, los trabajos del campo de la salud ocupacional deben contener la evaluación de los aspectos preventivos del deterioro de la salud, así como aquellos que aseguran un funcionamiento óptimo (Salanova, Martínez y Llorens, 2005). En este sentido, los modelos de estudios recientes de la salud psicosocial en el trabajo incluyen indicadores de malestar y de bienestar. Es decir, incorporan tanto el burnout, y en concreto sus variables centrales -Agotamiento Emocional y Cinismo-, como el engagement, a partir de sus variables centrales -Vigor y Dedicación-(Schauefeli et al., 2002).

Al respecto, en un trabajo reciente, se analizan las relaciones establecidas por las variables como elementos del bienestar en facultativos, reproduciendo el patrón observado en diferentes poblaciones (Bernabé, Martín-Aragón, Terol, Quiles, y Cabrera, 2011). El primero de ellos, el burnout, ha sido ampliamente estudiado y es entendido como una respuesta prolongada a estresores crónicos a nivel personal y relacional en el trabajo. Este padecimiento es determinado a partir de las dimensiones conocidas como agotamiento, cinismo e ineficacia profesional (Maslach, Schaufeli y Leiter, 2001). El segundo de ellos, el engagement hace referencia a un estado mental positivo de realización vinculado con el trabajo, caracterizado por vigor, dedicación y absorción (Schaufeli y Bakker, 2003). Ambos representarían categorías en un modelo circunflejo de emociones, caracterizado por la activación y el placer (Bakker, Rodríguez-Muñoz y Derks, 2012).

Por otro lado, tanto en el modelo reformulado de Demandas y Recursos Laborales de Demerouti et al. (2001), como en el propuesto por Bakker y Demerouti (2008) y el de Recursos, Experiencias y Demandas, impulsado por Salanova, Cifre, Martínez y Llorens (2009), los recursos personales tienen un papel relevante en la salud laboral de los trabajadores. En estos modelos, la autoeficacia (Bandura, 1977 ; 1999) ha mostrado ser un recurso personal, tanto en la etiología del burnout (Gil-Monte, 1999) como en el desarrollo del engagement (Xanthopoulou, Bakker, Demerouti, y Schaufeli, 2007). 
De acuerdo con la Teoría Social Cognitiva (Bandura, 1977, 1999), la creencia o el grado de seguridad que un sujeto tenga sobre sus propias capacidades autoeficacia- influirá en la percepción de estrés en las situaciones amenazantes. También, determinará el grado de empeño y la facilidad o dificultad del individuo en la consecución de la tarea, así como las reacciones emocionales que acompañen esta acción. De esta forma, la autoeficacia parece tener un rol determinante ante la elección de los objetivos y los logros alcanzados en el trabajo diario.

Los estudios al respecto parecen apoyar esta hipótesis. Así, por una parte, existe evidencia de que una exposición mantenida al estrés ocupacional y una baja percepción de eficacia para hacer frente a las demandas laborales incrementa la vulnerabilidad al burnout (Salanova, Peiró, y Schaufeli, 2002; Beas y Salanova, 2006; Heuven, Bakker, Schaufeli, y Huisman, 2006; Schwarzer y Hallum, 2008); y por otra parte, una fuerte percepción de eficacia mejora las respuestas de engagementen diferentes poblaciones profesionales (Heuven et al., 2006; Llorens, Salanova, Schaufeli y Bakker, 2007; Xanthopoulou et al. 2007).

Ahora bien, la perspectiva de este estudio se dirige a evaluar el papel de la autoeficacia como recurso personal en la salud laboral de los empleados. La novedad de este trabajo radica principalmente en la muestra evaluada, al tratarse de profesionales sanitarios, y en concreto de médicos facultativos de los servicios de emergencias y urgencias sanitarias. Al respecto, estos servicios pueden considerarse en sí mismos como hospitales en miniatura, con múltiples situaciones y escenarios a los que hacer frente (Montero, Calderón de la Barca, Jiménez, Berlango, Pérez et al., 2000); además, estos centros han estado expuestos a un cambio progresivo en su estructura, ajustándose a la organización de los servicios sanitarios, los cuales condicionan los horarios, sistema de turnos de trabajo, recursos disponibles y las funciones que se realizan.

Moreno et al. (2008) plantean que el uso de tales unidades se ha incrementado en España en un $80.7 \%$ en 11 años, reduciendo el porcentaje de ingresos, pero manteniendo la tasa de presión urgente por encima del $65 \%$. La sobrecarga existente es un problema común a otros países; respecto a la Medicina de Emergencias, tal como señala el American College of Emergency Physicians (Vickman, 2004), el volumen y la intensidad del trabajo son estresores que acompañan habitualmente la praxis en esta especialidad.

Por otro lado, una particularidad de estos centros médicos es la dificultad de coordinación entre las unidades, debido, en parte, a la falta de reconocimiento de 
la especialidad en Medicina de Emergencias, que permita agrupar el conjunto de conocimiento y procesos médico-asistenciales que se realizan en los SUH; esto dificulta la delimitación de las funciones y competencias de esta praxis médica.

Además de lo anterior, se ha observado un creciente cambio de expectativas en los ciudadanos con respecto a estas unidades y un uso indebido, a causa de la falta de conocimiento sobre los servicios sanitarios (SEMES, 2003). Esta consecuencia implica un incremento importante de la utilización de los SUH, así como una atención continuada ( 365 días del año, 7 días de la semana y 24 horas al día). Esto obliga la organización de turnos de trabajo de 8 horas y guardias de 24 horas en cada hospital, lo que exige, en la mayoría de casos, un número mínimo de guardias al mes. Si bien la realización de guardias por parte del médico es voluntaria, el profesional debe cumplir más jornadas laborales de las deseadas a fin de cumplir con las necesidades del servicio.

Bajo este contexto de trabajo, los modelos desde la Teoría Social Cognitiva (Bandura, 1977) otorgan a las variables del self un papel central para explicar el desarrollo del burnout. De acuerdo a estos modelos, la etiología del burnout desde la autoeficacia parte de los siguientes puntos: a) Las cogniciones de los individuos determinan su forma de percibir y de actuar; $b$ ) los efectos de las acciones de los propios sujetos y las consecuencias observadas a partir de las acciones de los demás, influencian a su vez estas cogniciones; y, c) la creencia o el grado de seguridad que un sujeto tenga sobre sus propias capacidades, determinará el grado de empeño y la facilidad o dificultad en la consecución de la tarea, así como las reacciones emocionales que acompañan a esta acción.

Según el Modelo de Competencia Social de Harrison (1983), la motivación a ayudar tiene un gran peso. Los médicos que trabajan en estos servicios están altamente motivados para ayudar a los demás. Por otra parte, al llevar a cabo la conducta, encuentran en su entorno laboral factores de ayuda y factores barrera para el desempeño. Los factores de ayuda son: objetivos realistas de trabajo, ajuste entre los valores personales y de la institución, y disponibilidad de recursos. Los factores barrera son: ambigüedad de rol, ausencia de recursos y sobrecarga laboral, etc. Aunado a ello, en médicos de los servicios de urgencias, se observa como la existencia de ambos factores, relacionados con el descanso, oportunidades para desarrollarse y un adecuado turno de trabajo se asocia con los niveles de eficacia profesional y satisfacción laboral (Bernabé, Martín-Aragón y Terol, 2013). 
De acuerdo al modelo, la motivación para ayudar de los facultativos va a determinar su eficacia en la consecución de los objetivos laborales, de forma que mientras mayor sea la motivación del trabajador, mayor será la eficacia laboral y se espera que tenga un mayor engagement. Por el contrario, la presencia de factores barrera disminuye la percepción de eficacia laboral, pues impide lograr los objetivos; además, en caso de mantenerse esa situación con el transcurrir del tiempo, incidiría en el desarrollo del burnout, incrementando la percepción de las barreras y la baja percepción de eficacia (Gil-Monte, 2005).

Según Harrison (1983) este modelo es especialmente aplicable a los trabajadores que han desarrollado expectativas muy elevadas sobre la consecución de sus objetivos laborales e intentan ayudar a los demás en situaciones que requieren algo más que la simple motivación para alcanzar el éxito. Este puede ser el caso de la muestra de estudio.

En los últimos años, el estudio del burnout en el ámbito sanitario se ha relacionado con la ausencia de determinados recursos como son el apoyo social, la posibilidad de control y la autonomía (Vegchel, Jonge, Soderfeldt, Dormann y Schaufeli, 2004; Cydulka y Korte, 2008; Wu, Zhu, Li, Wang y Wang, 2008), con la percepción de inequidad en la relación médico-paciente (Smets, Visser, Oort, Schaufeli y De Haes, 2004), o con la vivencia de incidentes críticos o estrés postraumático (van der Ploeg, Dorresteijn y Kleber, 2003; Reinhard y Maercker, 2004; Martín-Aragón, Quiles, Quiles, Esclapés y Bernabé, 2006). Respecto al engagement, solo encontramos trabajos en personal de enfermería, cuidadores formales y odontólogos (Schaufeli y Bakker, 2004; Hakanen, Bakker y Demerouti, 2005; Gorter, Brake, Hoogstraten y Eijkman, 2007), pero ninguno de ellos analiza el papel de las creencias de autoeficacia en médicos.

Por lo tanto, en este trabajo pretendemos, en primero lugar, explorar las relaciones entre la autoeficacia y la salud psicolaboral de los médicos de los servicios de urgencias hospitalarias, y en segundo lugar, comprobar un modelo donde sucede lo mismo que en otras muestras no sanitarias en las que se ha estudiado este efecto (Hakanen, Bakker y Schaufeli, 2005; Heuven et al., 2006). Es decir, que la autoeficacia explique los resultados en las respuestas de burnout -agotamiento emocional y cinismo-, y de engagement -vigor y dedicación-, en los facultativos de emergencias hospitalarias. 


\section{MÉTODO}

\section{Participantes}

Se solicitó la participación voluntaria de todos los facultativos de los Servicios de Urgencias Hospitalarias $(\mathrm{N}=117)$ de los ocho hospitales públicos de la provincia de Alicante, obteniendo una tasa de respuesta del $71.8 \%(n=84)$.

La edad media de la muestra de estudio fue de 40.45 años ( $\mathrm{dt}=7.79$ ); el $54.8 \%$ de los participantes eran hombres $(n=46)$, y el $53.6 \%$ estaban casados 0 vivían en pareja. Respecto al tipo de contrato, la mayoría de los facultativos tenía estabilidad laboral: el $25 \%$ era funcionario $(n=21)$ y el $36.9 \%$ ocupaba una plaza de interinidad $(n=31)$. El resto tenía contratos por acumulación de tareas $(23.8 \%$; $n=20)$ o estaban realizando una sustitución $(14.3 \% ; n=12)$. El tiempo medio de trabajo en los servicios de urgencias fue de 9.48 años $(\mathrm{dt}=6.84)$.

\section{Variables e instrumentos}

Cuestionario de variables sociodemográficas y laborales: se incluyó información sobre edad, género, estado civil o de convivencia, situación laboral y tiempo de trabajo en los servicios de urgencias hospitalarias.

Autoeficacia General: se utilizó la escala de autoeficacia general de Baessler y Schwarzer (1996), validada en la población española por Martín-Aragón et al. (2002). El cuestionario está compuesto por 10 ítems que recogen un sentimiento estable del individuo sobre su competencia personal para manejar de forma eficaz gran variedad de situaciones estresantes. La escala de respuesta es de tipo Likert, con 4 alternativas de respuesta referidas a la frecuencia de la creencia. La escala en nuestra muestra de estudio obtuvo una fiabilidad de .83 .

Salud psicosocial: se evaluaron las variables centrales del burnout-agotamiento y cinismo- y del engagement-vigor y dedicación. Para ello, se utilizaron las escalas de agotamiento emocional $(\alpha=.83)$ y cinismo $(\alpha=.79)$ del Maslach Burnout Inventory General Survey (MBI-GS: Schaufeli et al., 1996, adaptado a población española por Salanova et al., 2000). Las escalas están compuestas por 5 ítems y 4 ítems respectivamente. En cuanto a la evaluación de sentimientos positivos -vigor $(\alpha=.79)$ y dedicación $(\alpha=.81)$ - se utilizaron las escalas de la versión española del Utrech Work Engagement Survey (UWES; Schaufeli y Bakker, 2003). Están 
compuestas por 6 y 5 ítems respectivamente. La escala de respuesta es tipo Likert de 7 puntos ( 0 -nunca o ninguna vez-a 6 -siempre o todos los días).

\section{PROCEDIMIENTO}

Para la administración de las pruebas, se mantuvieron previamente entrevistas con los jefes de servicios de urgencias hospitalarias de los ocho hospitales públicos de la provincia de Alicante. Se acordaron diferentes días para la administración de la escalas durante la jornada laboral, coincidiendo con reuniones de sesiones clínicas. El investigador presentó los objetivos generales del estudio e informó sobre la confidencialidad de las respuestas y la voluntariedad de la participación. Una vez aceptada, los profesionales respondieron in situ el cuadernillo de preguntas. La tasa de respuesta fue del $71.8 \%(\mathrm{~N}=117)$.

\section{Análisis estadísticos}

En primer lugar se realizaron los análisis descriptivos, de fiabilidad y correlacionales para las escalas de autoeficacia general, y las subescalas de agotamiento emocional, cinismo, vigor y dedicación. Para el estudio de fiabilidad, se halló el Coeficiente Alfa de Cronbach, considerando satisfactorios los valores superiores a .70 (Nunally y Bernstein, 1978). Para el estudio de la relación entre las variables, se utilizó la correlación producto-momento de Pearson, siendo significativos los valores con un $p$-valor $<.01$ y .005 .

En segundo lugar, para el estudio del modelo propuesto se realizó un Análisis Factorial Confirmatorio, utilizando el programa AMOS 16 para determinar si los factores obtenidos se corresponden con los modelos previos acerca de los datos. Se tuvieron en cuenta como índices absolutos de ajuste, el estadístico $\chi^{2}$ así como los índices Goodness of Fit Index (GFI) (Jöreskog y Sörbom, 1986) y el Adjusted Goodness of Fit Index (AGFI). El rango de puntuaciones varía de 0 a 1, siendo indicativo de un buen ajuste valores próximos al límite superior. Respecto a los índices relativos se utilizó el Normed Fit Index (NFI) y el Comparative Fit Index (CFI) propuestos por Bentler y Bonnet (1980). Al igual que en los índices absolutos, valores próximos a 1 son indicativos de un mejor ajuste. Se considera un buen ajuste a los datos valores superiores a .90 . 
Por último, se utilizó el Root Mean Square Error of Approximation (RMSEA) como índice absoluto para valorar el residuo asociado al modelo, valores inferiores a .08 son indicativos de un buen ajuste, mientras que superiores a 1 implicaría el rechazo del modelo (Browne y Cudeck, 1993).

\section{RESULTADOS}

En la Tabla 1 se muestran los resultados de los análisis descriptivos de las escalas utilizadas, así como de las correlaciones. Todas las correlaciones son estadísticamente significativas.

\section{Tabla 1}

Análisis de consistencia interna y estadísticos descriptivos.

\begin{tabular}{lc|c|c|c|c|c|c|c|c}
\hline & Alfa & Rango & M & DT & $\mathbf{1}$ & $\mathbf{2}$ & $\mathbf{3}$ & $\mathbf{4}$ & $\mathbf{5}$ \\
\hline 1. Autoeficacia General & .83 & {$[1-4]$} & 3.07 & .35 & - & $-.45^{\star \star}$ & $-.37^{\star \star}$ & $.46^{\star \star}$ & $.42^{\star \star}$ \\
\hline 2. Agotamiento Emocional & .89 & {$[1-6]$} & 4.31 & 1.25 & & - & $.62^{\star \star}$ & $-.41^{* \star}$ & $-.34^{\star \star}$ \\
\hline 3. Cinismo & .83 & {$[1-6]$} & 4.19 & 1.21 & & & - & $-.46^{* \star}$ & $-.52^{\star \star}$ \\
\hline 4. Vigor & .79 & {$[1-5]$} & 2.69 & .74 & & & & - & $.70^{\star \star}$ \\
\hline 5. Dedicación & .81 & {$[1-5]$} & 3.07 & .34 & & & & & - \\
\hline
\end{tabular}

En la Figura 1 se muestra el resultado del modelo propuesto. Allí, puede observarse que la matriz de varianzas y covarianzas es estadísticamente significativa $\left(p<0,01 ; \chi^{2}: 118.2\right)$, así como todas las relaciones entre las variables del modelo estructural. Los índices de ajuste absoluto (GFI = .82; AGFI= .74) se encuentran próximos al valor 1 , al igual que los índices relativos (NFI = .78; CFI $=.88)$. El valor de índice absoluto es ligeramente superior al criterio propuesto (RMSEA = .09). 


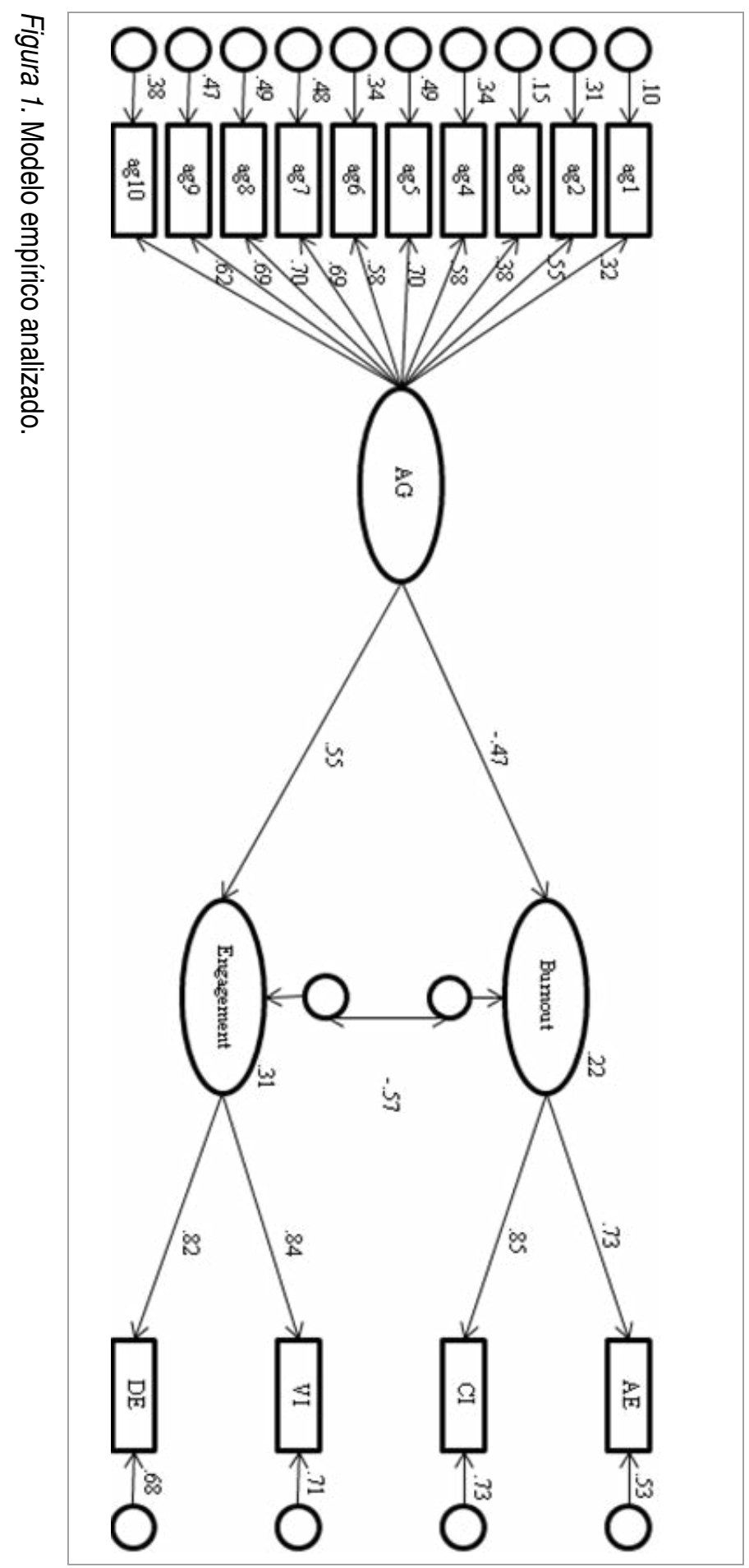




\section{DISCUSIÓN}

Con respecto a nuestro objetivo de explorar las relaciones entre la autoeficacia, entendida como un recurso personal, y la salud psicolaboral operativizada, mediante las variables centrales del burnout -agotamiento y cinismo- y del engagement -vigor y dedicación-, los resultados muestran relaciones significativas y en el sentido teórico esperado con cada una de ellas. Cuanta mayor es la creencia de los médicos de su propia capacidad para afrontar diferentes situaciones complicadas o problemáticas, menores son sus síntomas de agotamiento emocional y cinismo. Esto apoyaría la evidencia encontrada por otros autores, que al estudiar otras muestras han determinado que las bajas creencias de eficacia personal incrementan la vulnerabilidad al síndrome de burnout (Salanova et al., 2002; Beas y Salanova, 2006; Heuven, Bakker, Schaufeli, y Huisman, 2006; Schwarzer y Hallum, 2008). Asimismo, cuanto mayor es la creencia del facultativo en su propia capacidad para manejar diferentes situaciones problemáticas, mayores son las frecuencias de sus sentimientos de vigor y dedicación en el trabajo.

El sentido y la significación de esta relación también se ha encontrado en otros estudios realizados con muestras no sanitarias (Salanova, Bresó, y Schaufeli, 2005; Heuven et al., 2006; Llorens et al., 2007).

De acuerdo con la Teoría Social Cognitiva, los elevados niveles de autoeficacia en estos médicos pueden predecir el esfuerzo y la persistencia en su puesto de trabajo cuando se crean capaces de conseguirlo (Bandura, 1999). Una posible explicación a los elevados niveles de autoeficacia encontrados en los médicos del estudio puede deberse a las experiencias de dominio previas en las funciones del servicio, asociadas al logro de objetivos (por ejemplo atender una fuerte demanda de pacientes en múltiples ocasiones). Como se ha visto, las experiencias de dominio en situaciones particulares con éxito genera una robusta relación con las expectativas de autoeficacia (Bandura, 1977).

Con respecto al objetivo de probar un modelo, los índices de ajuste muestran un valor moderado, así como el resto de índices próximos al límite superior y al punto de corte según los criterios de Hu y Bentler (1999). El modelo determina que el número de factores obtenidos y sus cargas coinciden con lo esperado y observado en otras poblaciones. No obstante, estos resultados deben tomarse con cautela, debido a que los índices obtenidos no muestran un ajuste perfecto. Una posible explicación de un ajuste moderado puede deberse, en parte, al grado de generalidad de la Escala de Autoeficacia. En este sentido, aunque se 
ha demostrado que la tendencia general a sentirse eficaz puede extenderse a situaciones específicas (Yeo y Neal, 2006), en el dominio laboral, Beas y Salanova (2006) señalan mayor capacidad predictiva sobre el Burnout cuando se usan escalas específicas de autoeficacia. En la línea de Bandura $(1997 ; 1999)$ que definió la autoeficacia como un constructo específico que debe ser entendido de acuerdo a un nivel de competencia en una situación concreta, frente al mayor grado de generalidad defendido por Schwarzer (1992). Frente a esto, en el estudio optamos por una medida general, para poder de este modo en futuras investigaciones realizar comparaciones con diferentes grados de especificidad de la variable.

Los resultados alcanzados nos animan a orientar los futuros estudios hacia la inclusión de una medida específica para poder explorar con mayor profundidad y concreción la relevancia de las creencias de autoeficacia como recurso personal relevante, a fin de hacer frente a las demandas en el trabajo y experimentar sentimientos positivos que favorezcan el afrontamiento futuro, y de este modo, apoyar los resultados obtenidos en otras profesiones (Hakanen et al. 2005; Xanthopoulou et al., 2007).

Por tanto, y a pesar de la moderación de los resultados obtenidos, podemos decir que, en general, los datos se orientan hacia el apoyo de la propuesta de los modelos de estudio recientes de salud psicosocial en el trabajo, que señalan el papel determinante de los recursos personales en el desarrollo de tales respuestas de burnout y de engagement, en concreto el papel de la autoeficacia como variable que transformaría las emociones en el trabajo (Heuve et al., 2006). Así, la orientación de las intervenciones hacia la potenciación de la autoeficacia debe valorarse al momento de diseñar programas de promoción de la salud laboral en los médicos de urgencias hospitalarias.

En este sentido, los trabajos más recientes identifican la existencia de espirales de ganancia que reforzarían una respuesta de afrontamiento adaptativo, tal y como se recoge en el modelo propuesto por Llorens et al. (2007). Sería adecuado, por tanto, diseñar y evaluar un programa centrado en este aspecto para valorar su eficacia en la prevención de los síntomas de agotamiento y cinismo, y en el desarrollo de sentimientos y comportamientos relacionados con el vigor y la dedicación en el trabajo. 


\section{REFERENCIAS BIBLIOGRÁFICAS}

ANTONOVSKY, A. (1987). Unraveling the mystery of health. San Francisco: Jossey-Bass Publishers.

BÄSSLER, J., Y SCHWARZER, R. (1996). Evaluación de la autoeficacia: Adaptación española de la escala de autoeficacia general. Ansiedad y Estrés, 2(1), 1-8.

BANDURA, A. (1977). Self-efficacy: toward a unifying theory of behavioural change. Psychological Review, 83, 191-215.

BANDURA, A. (1999). Autoeficacia: Cómo afrontamos los cambios de la sociedad actual. Bilbao: Desclée De Brouwer.

BAKKER, A. B., Y DEMEROUTI, E. (2008). The crossover of work engagement: A closer look at the role of empathy. Journal of Managerial Psychology, 24(3), 220-236

BAKKER, A. B., RODRÍGUEZ-MUÑOZ, A., Y DERKS, D. (2012). La emergencia de la psicología de la salud ocupacional positiva. Psicothema, 24(1), 66-72

BEAS, M., Y SALANOVA, M. (2006). Self-efficacy beliefs, computer training and psychological well-being among information and communication technology workers. Computers in Human Behavior, 22, 1043-1058.

BENTLER, P. M. Y BONNET, D. G. (1980). Significance test and goodness of fit in the analysis of covariance structures. Psychological Bulletin, 88, 588-606.

BERNABÉ, M., MARTÍN-ARAGÓN, M., TEROL, M. C., QUILES, M. J. Y CABRERA, V. (2011). Análisis de algunos elementos de bienestar laboral en los médicos de los servicios de urgencias hospitalarios. Entre la ilusión y el agotamiento. Ansiedad y Estrés. 17(2-3), 221-231

BERNABÉ, M., MARTíN-ARAGÓN, M., TEROL, M. C., QUILES, M. J. (2013). Relaciones entre Estándares de Calidad de los Servicios de Urgencias Hospitalarios y la Satisfacción Laboral y la Eficacia Profesional de sus Facultativos. Pendiente de publicación. EMERGENCIAS. En revisión 
BROWNE, M. W., Y CUDECK, R. (1993). Alternative ways of assessing model fit. En K. A. Bollen y J. S. Longs (Eds.), Testing structural equation models (pp. 136162). Newbury Park, CA: Sage.

CYDULKA, R., Y KORTE, R. (2008). Career Satisfaction in Emergency Medicine: The ABEM Longitudinal Study of Emergency Physicians. Annals of Emergency Medicine, 51(6), 710-720.

GIL-MONTE, P. (1999). Perspectivas teóricas y modelos interpretativos para el estudio del síndrome de quemarse por el trabajo. Anales de Psicología, 15(2), 261-268.

GIL-MONTE, P. (2005). El síndrome de quemarse por el trabajo (Burnout): Una perspectiva histórica. En: Quemarse en el trabajo (pp. 37-71). Zaragoza: Egido Editorial.

GORTER, R., TE BRAKE, J., HOOGSTRATEN, J., Y ElJKMAN, H. (2007). Positive engagement and job resources in dental practice. Community Denistry \& Oral Epidemiology.

HARRISON, W. D. (1983). A social competence model of burnout. En B. A. Farber (Ed.), Stress and burnout in the human services professions. New York: Pergamon Press.

HAKANEN, J., BAKKER, A., Y DEMEROUTI, E. (2005). How dentists cope with their job demands and stay engaged: The moderating role of job resources. European Journal of Oral Sciences, 113, 487-497.

HAKANEN, J., BAKKER, A., Y SCHAUFELI, W. B. (2005). Burnout and work engagement among teachers. Journal of School Psychology, 43, 496-513.

HEUVEN, E., BAKKER, A., SCHAUFELI, W. B., Y HUISMAN, N. (2006). The role of self-efficacy in performing emotion work. Journal of vocational behaviour, 69 , 222-235.

HU, L. T., Y BENTLER, P. M. (1999). Cut off criteria for fit indexes in covariance structure analysis: Conventional criteria versus new alternatives. Structural Equation Modeling, 6, 1-55. 
JÖRESKOG, K. G., Y SÖRBOM, D. (1986). PRELIS: A program for multivariate data screening and data summarization: a pre-processor for LISREL. Moreesville, Ind. Scientific Software Inc.

LISBONA, A., Y PALACÍ, F. J. (2010). La medida de la iniciativa personal. Adaptación al castellano. Manuscrito presentado para su publicación.

LLORENS, S., SALANOVA, M., SCHAUFELI, W., Y BAKKER, A. (2007). Does a positive gain spiral of resources, efficacy beliefs and engagement exist? Computers in Human Behavior, 23, 825-841.

MARTÍN-ARAGÓN, M., PASTOR, M. A., CASTEJÓN, J, LLEDÓ, A., LÓPEZ-ROIG, S., TEROL, M. C. Y RODRÍGUEZ-MARÍN, J. (2002). Valoración preliminar de la Escala de Autoeficacia General en una muestra española. Revista de Psicología Social Aplicada, 12(2), 55-65.

MARTÍN-ARAGÓN, M., QUILES, M. J., QUILES, Y., ESCLAPÉS, C., Y BERNABÉ, M. (2006). Burnout en profesionales de las emergencias extrahospitalarias. Revista de Psicología Social, 16(3), 235-247.

MASLACH, C., SCHAUEFLI, W., Y LEITER, M. P. (2001) Job burnout. Annual Review of Psychology, 52, 397-422.

MONTERO, F., CALDERÓN DE LA BARCA, J., JIMÉNEZ, L., BERLANGO, A., Y PÉRULA, L. (2000). Situación actual de los Servicios de Urgencias Hospitalarios en España (I): Descripción general y análisis de la estructura física y funcional. Emergencias, 12, 226-236.

MORENO, M. (2008). ¿Y si adaptáramos los servicios hospitalarios de urgencias a la demanda social y no a las necesidades de salud? Emergencias, 20, 276-284.

NUNNALY, J. C., Y BERNSTEIN, I. H. (1994). Psychometric theory (3rd ed.). Nueva York: McGraw-Hill.

REINHARD, F., Y MAERCKER, A. (2004). Secondary traumatization, posttraumatic stress disorder, burnout, and social support in emergency care personnel. Zeitschrift fur Medizinische Psychologie, 13(1), 29-36. 
SEMES. (2003). Urgencias Sanitarias en España: Situación actual y propuestas de mejora. Granada: EASP.

SALANOVA, M., SCHAUFELI, W. B., LLORENS, S., PEIRÓ, J. M., Y GRAU, R. (2000). Desde el "Burnout" al "Engagement": ¿Una nueva perspectiva? Revista de Psicología del Trabajo y de las Organizaciones, 16(2), 117-134.

SALANOVA, M., PEIRÓ, J. M., Y SCHAUFELI, W. B. (2002). Self-efficacy specifity and Burnout among Information Technology Workers: An extension of the job Demands-Control Model. European Journal on Work and Organizational Psychology, 11, 1-25.

SALANOVA, M., BRESÓ, E., Y SCHAUFELI, W. (2005). Hacia un modelo espiral de las creencias de eficacia en el estudio del burnout y del engagement. Ansiedad y Estrés, 11, 215-231.

SALANOVA, M., MARTÍNEZ, I., Y LLORENS, S. (2005). Psicología Organizacional Positiva. En F. Palací (Ed.), Psicología de la Organización (pp. 349-376). Madrid: Pearson Prentice Hall.

SALANOVA, M., MARTíNEZ, I., CIFRE, E., LLORENS, S. (2009). La salud ocupacional desde la perspectiva psicosocial: aspectos teóricos y conceptuales. En M. Salanova, (Ed) Psicología de la Salud Ocupacional (pp. 27-56). Madrid: Síntesis

SELIGMAN, E., Y CSIKSZENTMIHALYI, M. (2000). Positive Psychology, an introduction. American Psychologist. 55, 5-14

SELIGMAN, E. (2008). Positive Health. Applied Psychology, 57(1), 3-18.

SCHAUFELI, W. B., MASLACH, C., JACKSON, S. E, LEITER, E. (1996). Maslach Burnout Inventory. Manual (2 ed.). Palo Alto, CA.: Consulting Psychologists Press.

SCHAUFELI, W. B., SALANOVA, M., GONZÁLEZ-ROMÁ, V., Y BAKKER, A. (2002). The measurement of burnout and engagement: a confirmatory factor analytic approach. Journal of Happiness Studies, 3, 71-92.

SCHAUFELI, W. B., Y BAKKER, A. (2003). The Utrecht Work Engagement Scale. Recuperado el 31/01/07, de http://www.schaufeli.net 
SCHAUFELI, W. B., Y BAKKER, A. (2004). Job demands, job resources, and their relationship with burnout and engagement: a multi-sample study. Journal of Organizational Behavior, 25, 293-315.

SCHWARZER, R., Y HALLUM, S. (1992). Self-Efficacy. Thought control of action. Bristol: Taylor \& Francis.

SCHWARZER, R., Y HALLUM, S. (2008). Pereceived Teacher Self-Efficacy as a Predictor of Job Stress and Burnout: Mediation Analyses. Applied Psychology: an international review, 57, 152-171.

SMETS, E., VISSER, M., OORT, F., SCHAUFELI, W. B., Y DE HAES, H. (2004). Perceived inequity: Does it explain burnout among medical specialists?. Journal of Applied Social Psychology, 34, 1900-1918.

VAN DER PLOEG, E., DORRESTEIJN, S., Y KLEBER, R. (2003). Critical incidents and chronic stressors at work: Their impact on forensic doctors. Journal of Occupational Health Psychology, 8(2), 157-166.

VEGCHEL, N., JONGE, J., SODERFELDT, M., DORMANN, C., Y SCHAUFELI, W. B. (2004). Quantitative Versus Emotional Demands Among Swedish Human Service Employees: Moderating Effects of Job Control and Social Support. International Journal of Stress Management, 11(1), 21-40.

VICKMAN, L. (2004). Stressors in Emergency Medicine. Burnout. En S. Bintliff, J. Kaplan y M. Meredith (Eds.), Wellness Book for Emergency Physicians. Dallas: American College of Emergency Physicians.

WU, S., ZHU, W., LI, H., WANG, Z., Y WANG, M. (2008). Relationship between job burnout and occupational stress among doctors in China. Stress and Health: Journal of the International Society for the Investigation of Stress, 24(2), 143-149.

XANTHOPOULOU, D., BAKKER, A., DEMEROUTI, E., Y SCHAUFELI, W. B. (2007). The role of Personal Resources in the Job Demands-Resources Model. International Journal of Stress Management, 14(2), 121-141.

YEO, G. B., Y NEAL, A. (2006). An examination of the dynamic relationship between self-efficacy and performance across levels os analysis and levels specifity. Journal of Applied Psychology, 91, 1088-1101. 